NISTIR 8174

\title{
Industrial Wireless Systems Workshop Proceedings
}

Richard Candell

This publication is available free of charge from:

https://doi.org/10.6028/NIST.IR.8174

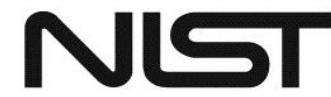

National Institute of Standards and Technology U.S. Department of Commerce 


\title{
Industrial Wireless Systems Workshop Proceedings
}

\author{
Richard Candell \\ Intelligent Systems Division \\ Engineering Laboratory
}

This publication is available free of charge from:

https://doi.org/10.6028/NIST.IR.8174

May 2017

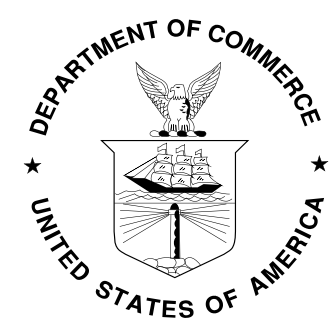

U.S. Department of Commerce

Wilbur L. Ross, Jr., Secretary

National Institute of Standards and Technology Kent Rochford, Acting NIST Director and Under Secretary of Commerce for Standards and Technology 


\section{Table of Contents}

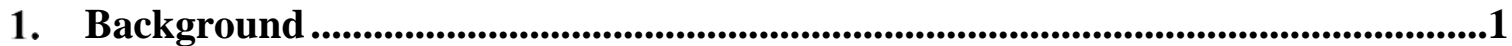

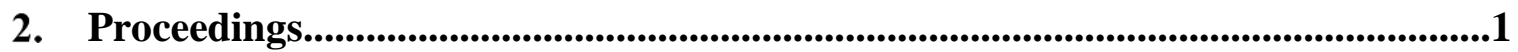

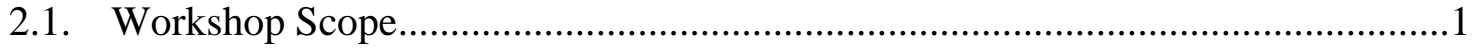

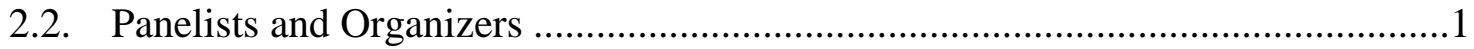

2.3. Workshop Report Reading Considerations .........................................................

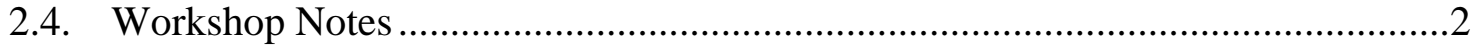

\section{NIST Disclaimer}

Certain commercial equipment, instruments, or materials are identified in this paper in order to specify the experimental procedure adequately. Such identification is not intended to imply recommendation or endorsement by the National Institute of Standards and Technology, nor is it intended to imply that the materials or equipment identified are necessarily the best available for the purpose. 


\section{Background}

Wireless technology has great appeal to many manufacturers, in this case industrial automation systems, which include process control, discrete manufacturing, safety systems, and building automation. Applying wireless sensing and control technologies in new or existing systems for monitoring and controlling equipment and processes eliminates costly cables, enables configuration flexibility, and can improve plant operating conditions, performance, and efficiency. Before applying these technologies, companies need to determine what wireless technologies would suitable for their application in a possibly challenging industrial radio frequency (RF) environment with many potential physical obstructions and sources of interference.

To help manufacturers make these kinds of decisions, the National Institute of Standards and Technology (NIST), the Institute of Electrical and Electronics Engineers (IEEE), and the IEEE Instrumentation and Measurement Society sponsored an Industrial Wireless Systems Workshop that was held on March 13, 2017. The workshop was hosted by Rowan University in Glassboro, NJ and more than thirty stakeholders from industry, academia, and government participated in the event. The results of the workshop discussions and agreements are provided in this proceedings document.

\section{Proceedings}

\subsection{Workshop Scope}

This workshop was held to explore the latest and future wireless technologies for establishing best practice guidelines to help manufacturers and other users make confident decisions in selecting and applying appropriate wireless technologies for their plants or factories based on their operating requirements and environments.

\subsection{Panelists and Organizers}

The following persons either organized the workshop, participated as a panel member, or both:

- From ABB: Zhibo Pang

- From Boeing: Al Salour, Bala Chidambaram, Mark Vanhorne

- From Phoenix Contact: Justin Shade

- From X8, LLC and ISA: Sterling Rooke

- From IEEE: Allen C. Chen, Kim Fung Tsang, Victor Huang, John Schmalzel

- From NIST: Richard Candell (Engineering Laboratory), Kang Lee (Engineering Laboratory)

\subsection{Workshop Report Reading Considerations}

The following considerations should be taken when reading the workshop output slides:

- In most cases, the wording is taken verbatim from the notes written on the whiteboards during the workshop.

- Text shown in brackets "[ ]" was added for clarity. The original point may be underlined to add emphasis on what was recorded. 


\subsection{Workshop Notes}

Notes from the workshop were captured as a slide presentation, and are included in the following pages. Table 1 lists acronyms used within the slides.

Table 1. List of Acronyms

\begin{tabular}{|l|l|}
\hline Acronym & Meaning \\
\hline ABB & The “ABB” corporation \\
\hline BYOD & Bring your own device \\
\hline IEEE & Institute of Electrical and Electronics Engineers \\
\hline IES & Industrial Electronics Society \\
\hline IMS & Instrumentation and Measurement Society \\
\hline ISA & International Society of Automation \\
\hline LLC & Limited Liability Coporation \\
\hline MTBF & Mean time between failure \\
\hline NIST & National Institute of Standards and Technology \\
\hline POC & Point of Contact \\
\hline Q\&A & Questions and answers \\
\hline RF & Radio frequency \\
\hline SIS & Safety integrated system \\
\hline
\end{tabular}




\section{NIST A IEEE leO :}

Industrial Wireless Systems Workshop

March 13, 2017

NIST/IEEE IMS/IEEE IES

Rowan University, Glassboro, NJ

POC: Richard Candell, NIST

rick.candell@nist.gov

\section{Workshop Summary}

Morning Session:

- Panelists gave short presentations

Afternoon Session:

- Panelist Questions \& Answers (Q\&A), Breakout/Brainstorming Sessions

- Breakout sessions were originally divided into guidelines and use cases groups. The groups were then combined because many attendees wanted to attend both group discussions.

Results:

- Consensus was Guidelines will be produced as a NIST document with standardization to be considered later.

- Participation by industry and academia is highly encouraged.

- Ad-hoc working group was recommended to be formed to develop a guidelines document. Consensus was NIST to take the lead on forming the working group. The working group will be established within two months of the workshop and will convene monthly.

- A group photo was taken 


\section{Organizers and Panelists}

ABB

- Zhibo Pang

Boeing

- Al Salour

- Bala Chidambaram

- Mark Vanhorne

Phoenix Contact

- Justin Shade

X8, LLC and ISA

- Sterling Rooke
IEEE

- Allen C. Chen

- Kim Fung Tsang

- Victor Huang

- John Schmalzel

NIST

- Rick Candell (Engineering Lab) rick.candell@nist.gov

- Kang Lee (Engineering Lab) wireless.lee@gmail.com

\section{Considerations when reading the notes}

For the Breakout/Brainstorming Session

- In most cases, the wording is taken verbatim from the notes written on the whiteboards

- Text in brackets "[ ]" was added for clarity. Original point may be underlined to add emphasis on what was actually recorded. 


\section{Breakout/Brainstorming Sessions}

Industrial Wireless Systems Workshop

\section{Use Cases and Issues}

Industrial Wireless Systems Workshop 


\author{
Breakout Session Discussions \\ Issues for Using Industrial Wireless in Factories
}

- Power/battery

- Multipath

- Noise

- Latency

- Security

- Life cycle costs

- Bandwidth

- Throughput

- Scalability

- Device cost

- Ease of use
- Interoperability

- Co-existence

- Size

- Integrability

- Environmental reliability

- MTBF [monitoring machine health]

- Safety

- Intrinsic safety

- Maintainability

- Legacy Interfaces (analog)

Breakout Session Discussions

Critical Success Factors

- [Does it improve] product quality?

- [Does it improve] product cost?

- [Does it improve human] safety?

- [Does it increase] production system reliability?

- [Does it improve evidence of] regulatory compliance?

- [Does it enhance] environmental stewardship (waste, pollution, radiation, clean air)? 
Breakout Session Discussions

Types of Wireless Systems and Uses

- Monitoring, alarm, safety monitoring

- Supervisory

- Feedback

- Operational

- Materials/inventory management

- Identification

- Localization

- SIS (Safety Integrated systems)

- RF Channel diagnostics (channel sounding)
- Hand-held detector of emergency equipment within the cabin of aircraft.

- Automated paint stackers

- Identify unauthorized staff in safety zones

- Taxonomy of types of industrial systems could be helpful

\section{Breakout Session Discussions \\ Factory Floor Example Use Cases}

- Gas detection for confined spaces

- Scheduling of machines

- "In-situ" monitoring

- Human safety (Fall protection-heights, slips)

- Machine health monitoring

- Wireless dimensional probes (quality)

- Wing positioning for fuselage joining (quality)
- Hand-held detector of emergency equipment within the cabin of aircraft.

- Automated paint stackers

- Identify unauthorized staff in safety zones

- Taxonomy of types of industrial systems could be helpful 


\title{
Guidelines
}

Industrial Wireless Systems Workshop

\author{
Breakout Session Discussions \\ Suggested Document Sections \#1
}

- Existing \& Future wireless technologies

- Technology categories

- Technology selection

- Spectrum selection

- RF concerns

- Environment

- Site surveys

- Spectrum selection

- Policy/Procedures for "Bring Your Own Devices" (BYOD)

- Future capabilities \& concerns

- Spectrum monitoring/forecasting 
Breakout Session Discussions

Suggested Document Sections \#2

- Validation of new systems

- Tools/methods for testing

- Calibration of wireless sensors (analog)

- Acceptance plan for deployment

- Human Health \& Safety

- Medical Device compatibility

\author{
Breakout Session Discussions \\ Suggested Document Sections \#3
}

- Control [i.e. supervisory control]

- Monitoring [for accurate and timely observation of the process]

- Feedback [for machine control]

- Operations [to improve quality and productivity]

- refer to value proposition slide 
Breakout Session Discussions

Use of [Guidelines]Documents

- Used for education internally [within companies]

- Used to give to a supplier [for informational, requirements, and supplier conformance purposes]

- Best Practices [for selection and deployment of wireless systems]

- Spur Innovation [within the industrial wireless sector]

- Version Control [of the document assuming that this becomes a living document to be maintained over time.]

INTENDED DUE DATE 9/30/2018

\section{Next Steps}

Industrial Wireless Systems Workshop 


\section{Working Group and Guidelines Preparation}

- The consensus was that an ad-hoc working group is to be formed and led by NIST to develop NIST Guidelines on Industrial Wireless Systems

\section{- Action items:}

$>$ Victor Huang (IEEE IES) will email the raw notes taken at the workshop to Rick

Rick Candell (NIST) will prepare the notes for release

Rick Candell (NIST) will prepare a public announcement that a working group is to be formed to develop best practices for selection and deployment of wireless technologies for industrial environments.

$>$ Monthly working group meetings was agreed upon to make steady progress.

A mailing list will be created for interested parties.

\section{Group Photograph}

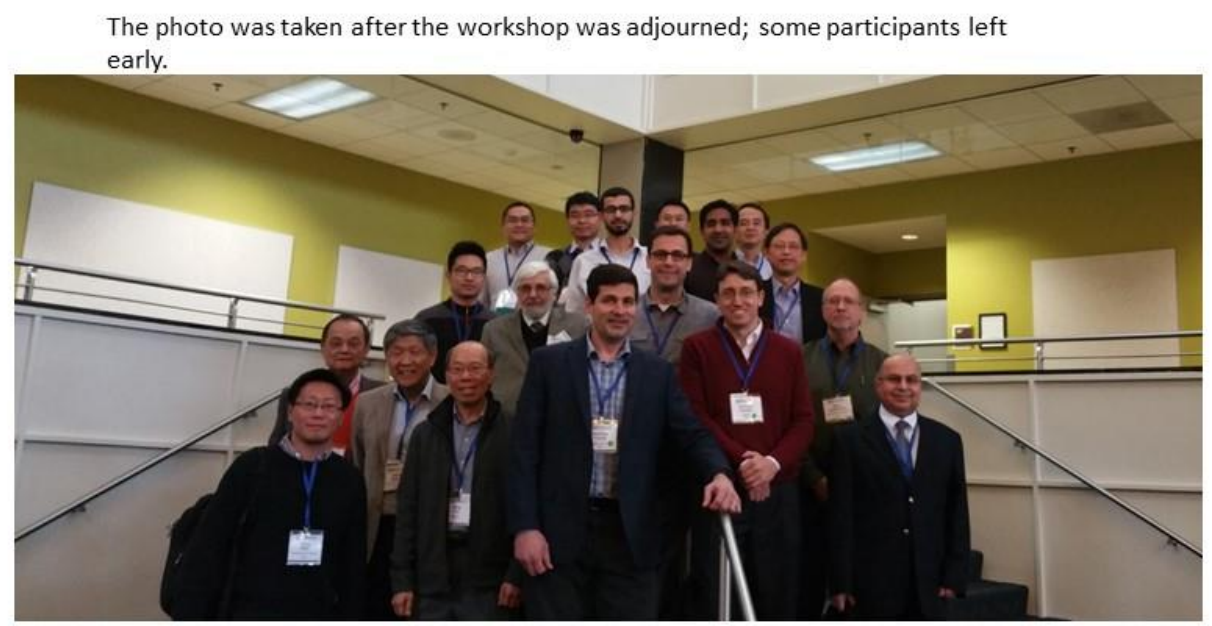

